\title{
ARTICLE OPEN \\ Selection of diverse strains to assess broad coverage of the bivalent FHbp meningococcal B vaccine
}

\author{
Shannon L. Harris ${ }^{1}$, Cuiwen Tan ${ }^{1}$, John Perez ${ }^{2}$, David Radley ${ }^{2}$, Kathrin U. Jansen (D) $^{1}$, Annaliesa S. Anderson ${ }^{1 *}$ and Thomas R. Jones ${ }^{1}$
}

MenB-FHbp is a recombinant meningococcal serogroup B (MenB) vaccine composed of 2 factor $\mathrm{H}$ binding proteins (FHbps). Meningococcal vaccines targeting polysaccharide serogroup $A, C, Y$, and $W$ capsules were licensed upon confirmation of bactericidal antibody induction after initial efficacy studies with serogroup $A$ and $C$ vaccines. Unlike meningococcal polysaccharide vaccines, wherein single strains demonstrated bactericidal antibodies per serogroup for each vaccine, MenB-FHbp required a more robust approach to demonstrate that bactericidal antibody induction could kill strains with diverse FHbp sequences. Serum bactericidal assays using human complement were developed for 14 MenB strains, representing breadth of meningococcal FHbp diversity of $\sim 80 \%$ of circulating MenB strains. This work represents an innovative approach to license a non-toxin protein vaccine with 2 antigens representing a single virulence factor by an immune correlate, and uniquely demonstrates that such a vaccine provides coverage across bacterial strains by inducing broadly protective antibodies.

npj Vaccines (2020)5:8; https://doi.org/10.1038/s41541-019-0154-0

\section{INTRODUCTION}

Although transmission of Neisseria meningitidis usually results in asymptomatic colonization of the upper respiratory tract, in some individuals, bacteremia and invasive meningococcal disease (IMD) occur. ${ }^{1-3}$ IMD commonly presents as meningitis and/or septicemia; pneumonia, septic arthritis, epiglottitis, and otitis media are less frequently observed. ${ }^{4,5} \mathrm{~A}$ high case fatality rate is associated with IMD (10-15\%), 6,7 and $20 \%$ of survivors have serious life-long sequelae such as limb amputation, hearing loss, and neurologic impairment. 8,9

Nearly all meningococcal disease worldwide is caused by 6 of the 12 characterized meningococcal serogroups (ie, A, B, C, W, X, and $Y) .^{10-12}$ Effective vaccines based on capsular polysaccharides have been developed for serogroups A, C, W, and $Y^{2}{ }^{2}$ However, immunogenicity of the MenB polysaccharide is poor because of similarity to polysialic acid structures present on human neuronal cells. ${ }^{13,14}$ During recent years, meningococcal serogroup B (MenB) in particular has been associated with a large proportion of IMD in Europe, ${ }^{15}$ the United States, ${ }^{16}$ Canada $_{1}{ }^{17}$ Australia, ${ }^{18}$ and New Zealand. ${ }^{19}$ Although vaccines based on outer membrane vesicles (OMVs) have been successfully used to control epidemics caused by a single MenB outbreak strain, ${ }^{20,21}$ the generated immune response is predominantly against the highly variable porin $A$ protein (PorA)..$^{2-25}$ Therefore, effectiveness is generally limited to the target strain. Consequently, surface-exposed proteins capable of inducing protective bactericidal antibodies across diverse MenB strains have been sought for the development of a broadly effective MenB vaccine.

Factor H binding protein (FHbp; also known as LP2086 and GNA1870), a conserved surface-exposed lipoprotein expressed on nearly all strains of MenB, was identified as such a target. ${ }^{26-31}$ Based on amino acid sequence, FHbp variants segregate into 2 immunologically distinct subfamilies (termed subfamily $A$ and subfamily B); each MenB strain expresses a single subfamily variant (Fig. 1a). ${ }^{32,33}$

MenB-FHbp (Trumenba ${ }^{\circledR}$, bivalent rLP2086; Pfizer Inc, Philadelphia, PA, USA) is a bivalent, recombinant protein MenB vaccine composed of equal amounts of 2 recombinant lipidated $\mathrm{FHbp}$ antigens, one from subfamily $A$ (variant A05) and the other from subfamily $B$ (variant B01). ${ }^{34}$ Importantly, it is predicted that this combination of $\mathrm{FHbp}$ variants is capable of providing protection against diverse MenB strains. ${ }^{33,35}$ MenB-FHbp has been approved for the prevention of IMD in several countries and regions, including the United States, Canada, Europe, and Australia. ${ }^{34,36-38}$ Another MenB vaccine, MenB-4C (Bexsero ${ }^{\circledR}, 4$ CMenB; GlaxoSmithKline Vaccines, Srl, Siena, Italy), also has a recombinant FHbp component (nonlipidated variant 1.1 from subfamily B) as well as 2 other recombinant protein antigens and an OMV. ${ }^{39}$ Thus, MenB-4C is different from MenB-FHbp, which contains two variants of a single antigen to afford broad coverage. ${ }^{40}$

The serum bactericidal assay using human complement (hSBA) measures complement-dependent, antibody-mediated lysis of meningococcal bacteria. An hSBA titer is defined as the highest serum dilution killing $\geq 50 \%$ of assay bacteria; ${ }^{41}$ an hSBA titer $\geq 1: 4$ is the accepted correlate of protection against meningococcal disease, ${ }^{41-43}$ and hSBA response rates based on this correlate have been used as surrogates for meningococcal vaccine efficacy. ${ }^{43}$ The SBA response rate has been specifically correlated with natural protection for the serogroup $C$ and $A$ polysaccharide vaccines. ${ }^{41}$ Because serogroup-specific polysaccharides are not variable, a single strain from each serogroup was sufficient to infer broad vaccine coverage. MenB OMV vaccines are also efficacious and vaccine-elicited $\mathrm{hSBA}$ titers correlated with protection against the target strain causing the epidemic. ${ }^{44-46}$ Accurately predicting strain coverage of protein-based vaccines is more complex using hSBA than for vaccines targeting capsular polysaccharides, given that protein sequence diversity and variability in expression levels differ among the different meningococcal disease strains. ${ }^{47}$ For example, PorA is the predominant target for serum bactericidal antibodies conferring protection after OMV vaccine immunization. ${ }^{48,49}$ PorA is a cell surface porin whose small cell surface exposed region has a high degree of sequence diversity. It has been estimated that protective immunity would need to be demonstrated with strains expressing 20 different PorA

\footnotetext{
${ }^{1}$ Pfizer Vaccine Research and Development, Pearl River, NY, USA. ${ }^{2}$ Pfizer Vaccine Research and Development, Collegeville, PA, USA. *email: Annaliesa.Anderson@pfizer.com
} 
a

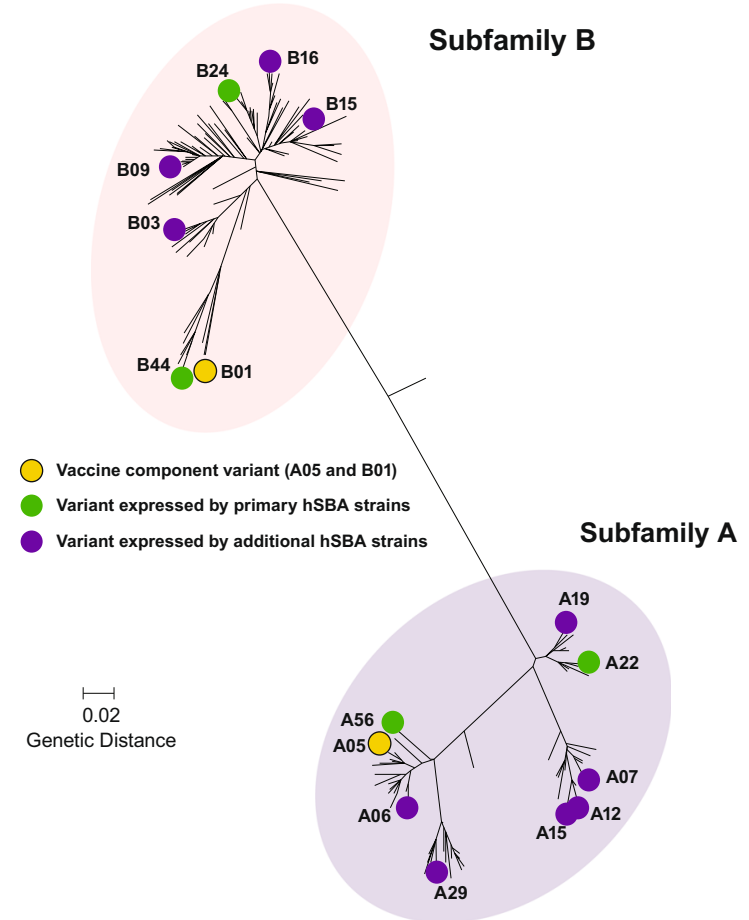

b

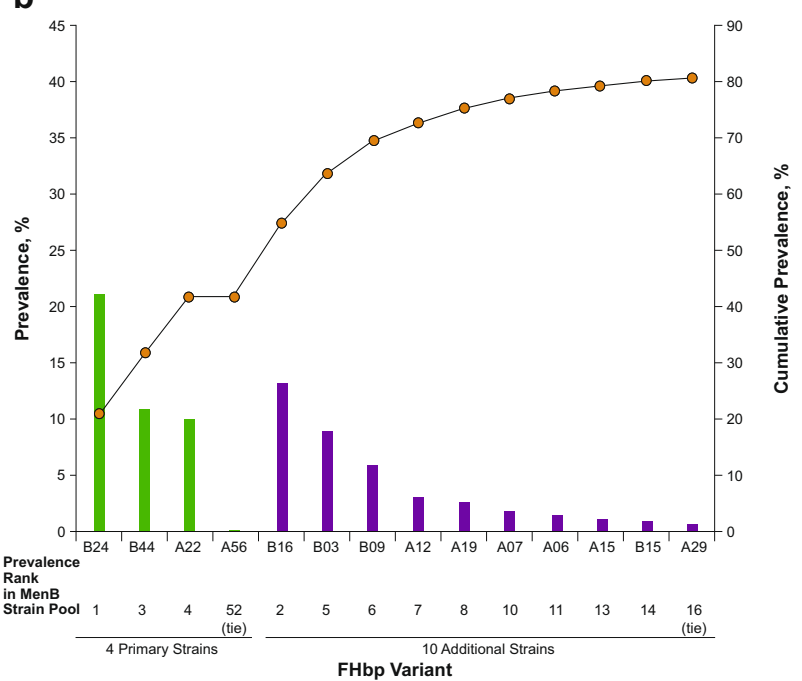

Fig. 1 Factor $\mathrm{H}$ binding protein (FHbp) phylogenetic tree: Primary and additional Neisseria meningitidis serogroup B (MenB) test strain variants ${ }^{32}$ and variant prevalence of primary and additional MenB test strains. In a, the phylogenetic and FHbp subfamily relationship of the FHbp variants expressed by the four primary and 10 additional MenB test strains is illustrated. The scale bar indicates genetic distance based on protein sequence. The amino acid sequence identity within FHbp subfamilies is $\geq 83 \%{ }^{33} \mathrm{hSBA}=$ serum bactericidal assay using human complement. Adapted from Ostergaard, L. et al. A bivalent meningococcal $B$ vaccine in adolescents and young adults. N. Engl. J. Med. 377, 2349-2362 (2017). In b, variant prevalence (left vertical axis; bars) and cumulative prevalence (right vertical axis; circles) are based on the MenB isolate collection $(n=$ 1263). Variants are ordered based on their prevalence rank in the MenB SBA isolate collection. Note that scales are different between left and right $y$-axes. SBA serum bactericidal assay.

serosubtypes to protect against approximately $80 \%$ of sporadic MenB disease-causing strains in the United States. ${ }^{50}$ Historically, OMV vaccines have contained 1 PorA and have not demonstrated protection against strains with PorA sequences that are heterologous in amino acid sequence compared with the vaccine antigen. ${ }^{51}$ Therefore, selection of representative test strains to demonstrate that vaccine-elicited antibodies can be effective against a meningococcal disease strain is of paramount importance for protein-based vaccines.

Immune sera elicited by MenB-FHbp in preclinical and early clinical studies demonstrated broad bactericidal antibodies that could kill diverse MenB strains containing FHbp subfamily A and B variants heterologous to the vaccine $\mathrm{FHbp}$ variants $\mathrm{A05}$ and B01. ${ }^{35,52,53}$ In an early assessment of the potential breadth of MenB-FHbp coverage, 100 MenB isolates with diverse FHbp variants, geographic origins, and genetic backgrounds were tested in hSBAs using MenB-FHbp immune rabbit serum. ${ }^{27,35}$ Of the 100 strains tested, 87 were killed in these hSBAs. ${ }^{35}$ Analysis of the 13 strains that were not killed suggested that the FHbp surface expression level on a given MenB strain affected the hSBA response. A threshold $\mathrm{FHbp}$ surface expression level was subsequently determined, above which isolates were predictably killed in hSBA. $^{54}$ Additional investigations of potential factors determining strain susceptibility found that killing was largely independent of FHbp sequence variant, multilocus sequence type, or PorA subtype. ${ }^{27,35}$

To select strains with broad antigenic and epidemiologic diversity for clinical testing, over 1200 invasive MenB disease isolates were collected from laboratories and health agencies in the United States and Europe to represent the prevalence of MenB isolates that were contemporary at the time of collection; all strains contained the FHbp gene. ${ }^{33}$ An unbiased approach was used to select four antigenically and epidemiologically diverse representative test strains for use in MenB-FHbp immunogenicity studies. ${ }^{27}$ Selection criteria included expression of $\mathrm{FHbp}$ variants heterologous to the vaccine antigens and adequately reflecting the diversity of $\mathrm{FHbp}$ in MenB disease isolates, low to medium FHbp surface expression levels, and low baseline hSBA seropositivity rates. These four primary MenB test strains express FHbp variants from both FHbp subfamilies (strain [variant]: PMB2001 [A22], PMB80 [A56], PMB2707 [B24], and PMB2948 [B44]; Fig. 1). ${ }^{32}$

To supplement immunogenicity data generated using the four primary MenB test strains and to demonstrate that immune responses against the four primary MenB test strains are predictive of immune responses against the diversity of $\mathrm{FHbp}$ variants expressed by MenB disease-causing isolates, hSBAs using 10 additional test strains were developed. The 10 additional test strains were selected to include prevalent $\mathrm{FHbp}$ variants found in MenB disease-causing strains in the United States and Europe. Here, we (i) describe the strategy and criteria used to select the 10 additional test strains, and (ii) present data demonstrating that the immune responses measured by hSBA using the four primary MenB strains are predictive of the responses obtained using 10 additional test strains, which further demonstrate and support the broad coverage of the immune response elicited by MenB-FHbp.

\section{RESULTS}

Sources and selection criteria for the additional MenB test strains Nine of the 10 additional MenB test strains were obtained from a collection of 1263 invasive disease-causing MenB strains (the MenB isolate collection). ${ }^{27,33}$ For the MenB isolate collection, US strains were from the Active Bacterial Core Surveillance sites (2000-2005), covering $\sim 13 \%$ of the population. European isolates (2001-2006) were from the public health laboratories of Norway, France, Czech Republic, and the Health Protection Agency in Manchester (which covers England, Wales, and Northern Ireland) and were collected systematically (every seventh or eighth isolate was included by order received at the country's reference laboratory) and represented $\sim 13 \%$ of invasive MenB isolates 
during the period. ${ }^{33,55}$ The strains expressing $\mathrm{FHbp}$ variant $\mathrm{A} 07$ were obtained from an extension of the MenB isolate collection that included an additional 551 disease-causing MenB strains from Spain and Germany $(n=1814)$. The extended MenB isolate collection was used as A07-expressing strains in the MenB isolate collection were not suitable because of the low surface expression of FHbp on these strains, high baseline seropositivity, and lack of readily available source of complement.

The criteria used to select the additional MenB test strains were (i) FHbp variant prevalence among MenB disease-causing strains in the United States and/or Europe, (ii) the FHbp variant needed to be different from those expressed by MenB primary test strains, (iii) in vitro FHbp expression levels at or below median levels for the respective $\mathrm{FHbp}$ variant group to ensure that the strain was representative of the variant group it belonged to, (iv) technical compatibility in the hSBA, and ( $\mathrm{v}$ ) being considered a predominant clonal complex for the variant group (if a predominant complex existed). Strains meeting these criteria also needed to be technically compatible in the hSBA, including adequate availability of suitable human complement lots (Fig. 2). Strains in each FHbp variant group with expression levels below the cutoff level (i.e., at or below median levels for the respective $\mathrm{FHbp}$ variant group) were randomly selected, with the first strains within an FHbp variant group meeting the required genetic, phenotypic, and hSBA development criteria becoming the additional MenB test strains. An exception to this methodology was made for the strain expressing $\mathrm{FHbp}$ variant B03, which was selected in collaboration with, and using guidance provided by, the US FDA based on its previous use in a phase 2 study. ${ }^{52}$

Characteristics of the additional MenB test strains

The 10 additional selected MenB test strains express FHbp variants A06, A07, A12, A15, A19, A29, B03, B09, B15, and B16 which differ from the ones in the four primary test strains (A22, A56, B24, B44) and have different sequences compared to the vaccine antigens (Table 1). The specific variants expressed by the four primary test strains are present in $42.0 \%(530 / 1263)$ of disease-causing isolates in the MenB isolate collection, and the specific variants expressed by the 10 additional test strains are present in an additional, non- overlapping $38.8 \%(490 / 1263)$ of disease-causing isolates in the MenB isolate collection (Fig. 1b).

Immunogenicity analysis: subjects with hSBA titer $\geq$ LLOQ for the 10 additional strains

The four primary strains were used to assess serological responses after two or three doses of MenB-FHbp in subjects participating in two pivotal phase 3 studies in adolescents and young adults. ${ }^{32}$ Serological responses to the 10 additional hSBA strains were assessed in a subgroup of the study subjects. The majority of subjects had hSBAs $\geq$ lower limit of quantitation (LLOQ; i.e., hSBA titer equal to $1: 8$ or 1:16, depending on strain) 1 month after dose 2 and 1 month after dose 3 for each of the primary $(64.0-99.1 \%$ and $87.1-99.5 \%$, respectively) and the 10 additional MenB test strains (51.6-100.0\% and 71.3-99.3\%, respectively) (Table 2). For the primary and additional MenB test strains, a substantial increase from baseline in the proportion of subjects achieving an hSBA titer $\geq$ LLOQ was observed among MenB-FHbp recipients (0, 2, 6 month schedule) after the second MenB-FHbp dose, with additional increases after the third dose.

Positive predictive values for the primary and additional strains The relationship between vaccine-induced hSBA responses for the primary MenB test strains and the 10 additional MenB test strains was assessed (Table 3). Within an FHbp subfamily, positive predictive values (PPVs) were greater than $80 \%$ for most primary/additional strain pairs 1 month after dose 3 . Thus, the immune responses measured in hSBAs using the primary test strains were highly predictive of immune responses for the additional strains within the same subfamily. The PPVs 1 month after dose 2 usually were slightly lower than those observed 1 month after dose 3 and ranged from $61.6 \%$ to $100 \%$ and $70.0 \%$ to $100 \%$ for subfamily A and B strain pairs, respectively, across studies. In summary, all PPVs showed high predictability for protective responses when comparing the primary and additional strain hSBA responses.

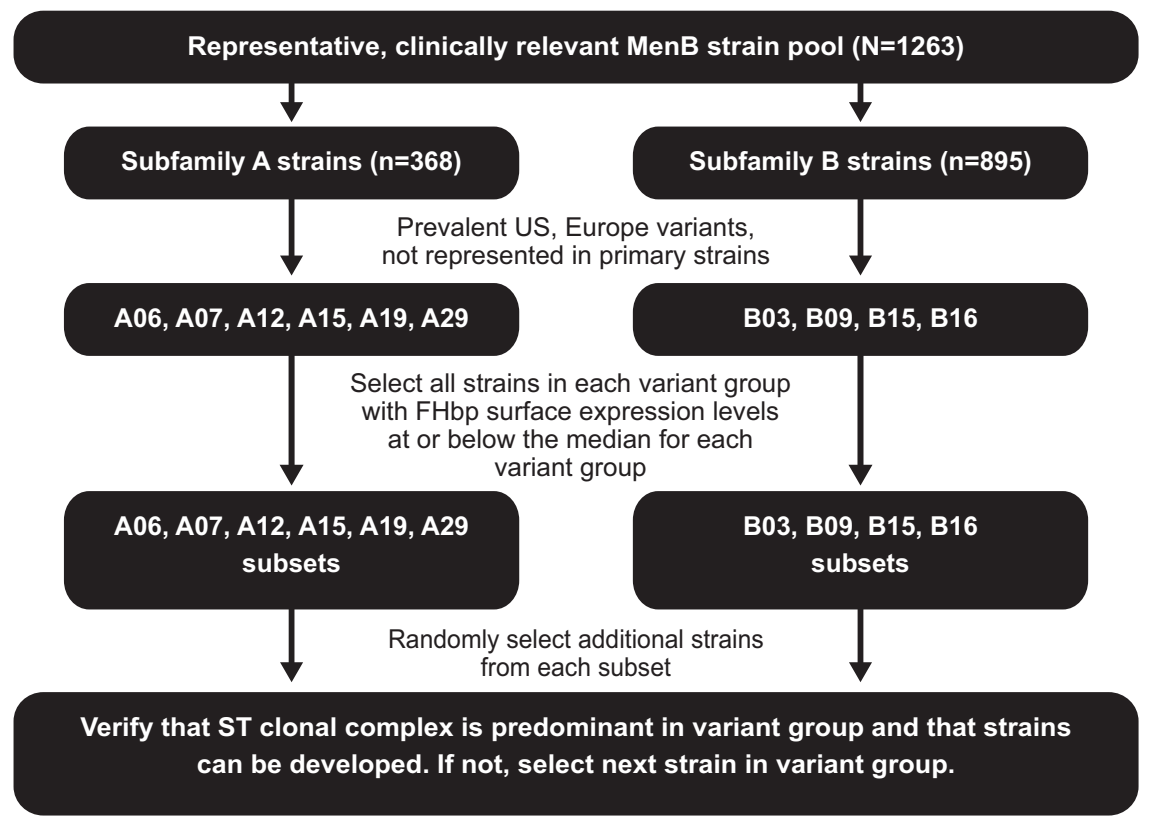

Fig. 2 Algorithm used for the selection of additional Neisseria meningitidis serogroup B (MenB) test strains. The MenB serum bactericidal assay (SBA) isolate collection $(n=1263)$ is used as the example in this figure. FHbp (factor H binding protein), ST (sequence type). 
S.L. Harris et al.

Table 1. Characteristics of the 4 Primary and 10 Additional MenB Test Strains.

\begin{tabular}{|c|c|c|c|c|c|c|}
\hline Strain & $\begin{array}{l}\text { FHbp } \\
\text { variant }\end{array}$ & $\begin{array}{l}\text { Percentage identity to } \\
\text { vaccine component }\end{array}$ & $\begin{array}{l}\text { Strain MEASURE MFI } \\
( \pm 1 \text { SD })\end{array}$ & $\begin{array}{l}\text { FHbp variant group MEASURE } \\
\text { MFI median }{ }^{b}( \pm 1 \text { SD })\end{array}$ & $\begin{array}{l}\text { Clonal } \\
\text { complex }\end{array}$ & $\begin{array}{l}\text { Country of } \\
\text { isolation }\end{array}$ \\
\hline \multicolumn{7}{|c|}{ Primary Strains } \\
\hline PMB2001 & A56 & 98.1 & $5002(3903,6410)$ & $5002^{c}$ & CC213 & France \\
\hline PMB2948 & B24 & 86.2 & $6967(5436,8929)$ & $8457(6599,10,839)$ & CC32 & France \\
\hline PMB3010 & A06 & 96.2 & $3370(2629,4319)$ & $3088(2410,3958)$ & CC461 & United Kingdom \\
\hline PMB3040 & $\mathrm{A} 07$ & 85.4 & $1379(1076,1767)$ & $1100(858,1409)$ & CC162 & Germany \\
\hline PMB824 & $\mathrm{A} 12$ & 85.4 & $2540(1982,3255)$ & $2467(1925,3161)$ & CC35 & United States \\
\hline PMB1672 & A15 & 85.1 & $2995(2337,3838)$ & $2904(2266,3721)$ & CC103 & France \\
\hline PMB1989 & A19 & 88.1 & $1934(1509,2479)$ & $1759(1372,2254)$ & $\mathrm{CC} 8$ & United Kingdom \\
\hline PMB648 & B16 & 86.2 & $2347(1831,3008)$ & $1996(1557,2558)$ & CC41/44 & United Kingdom \\
\hline \multicolumn{7}{|c|}{$\begin{array}{l}\text { FHbp factor H binding protein, MenB Neisseria meningitidis serogroup B, MFI mean fluorescence intensity, SBA serum bactericidal assay } \\
\text { a MFI } \pm 1 \text { SD from MEASURE assay } \\
\text { bBased on the MenB SBA isolate collection ( } n=1263 \text { ), except for variant group A07, which was calculated from the extended MenB SBA isolate collection } \\
\text { ( } n=1814 \text { ). Strains in each FHbp variant group with expression levels at or below median levels for the respective FHbp variant group were randomly selected. } \\
\text { The cutoff level adopted for each FHbp variant group was the observed median MFI plus } 1 \text { SD, using the precision estimate of } 25.2 \% \text { relative SD } \\
\text { 'There is only one strain expressing A56; thus, no SD values are included }\end{array}$} \\
\hline
\end{tabular}

\section{DISCUSSION}

A critical component of the clinical evaluation of the MenB-FHbp vaccine to determine the breadth of protection was the development of hSBAs using test strains with surface protein antigens whose sequence and expression variability are representative of the diversity of MenB disease-causing strains that were contemporary at the time of collection. As described in phase 3 studies in adolescents and young adults, hSBA response data for the four primary MenB test strains, all of which express $\mathrm{FHbp}$ variants heterologous to the vaccine antigens, strongly suggest that the bivalent MenB-FHbp vaccine provides broad coverage across diverse, disease-causing meningococcal strains. The 10 additional MenB test strains described here provide supportive immunologic data for MenB-FHbp and further confirm the validity of the use of the four primary test strains to measure the immune response to MenB-FHbp. As the responses obtained for the four primary test strains are predictive of the responses obtained for the additional 10 test strains, the immunological responses obtained by assessing the primary strains in hSBAs are representative of the diversity of strains causing invasive MenB disease.

For the hypothesis test-driven immunogenicity evaluations in licensure studies for MenB-FHbp, an unbiased approach was used to select the four primary MenB test strains from panels of diseasecausing MenB collected in the United States and Europe. A similar method was used to select the 10 additional MenB hSBA test strains, taking into consideration specific selection criteria to ensure that test strains were representative of the antigenic diversity of MenB isolates.

Collectively, the 14 MenB test strains represent the majority of the prevalent meningococcal $\mathrm{FHbp}$, with $\mathrm{FHbp}$ variants corresponding to $\sim 80 \%$ of circulating invasive disease-causing isolates in the United States and Europe.

Positive predictive value analyses were used to determine the association of immune responses, measured by hSBA, among primary and additional test strains expressing FHbps within the same subfamily. All of the PPV analyses showed the high predictability of the protective responses against the primary strain for the protective responses observed against the additional strains. These PPV analyses indicate that the responses observed against the four primary MenB test strains are representative of responses to other disease-causing MenB strains that express additional sequence-diverse $\mathrm{FHbp}$ variants different from the vaccine antigen variants.

The MenB-FHbp-elicited responses measured by hSBA to the four primary and 10 additional MenB test strains were evaluated using sera from individual vaccine recipients. By determining the proportion of vaccinated subjects with functional bactericidal antibodies, assessment of the breadth of MenB-FHbp coverage at the individual level was determined, which is not possible using pooled sera. The four primary MenB test strains were selected to represent the diversity of MenB disease-causing IMD and thus support the potential breadth of coverage for MenB-FHbp using hSBA. $^{47}$ Responses of individuals with hSBA titers $\geq 1: 4$ are the accepted correlate of protection and a surrogate of meningococcal vaccine efficacy. ${ }^{41-43,56}$ Thus, the responses provide a comprehensive and biologically predictive assessment of breadth of vaccine coverage. The relevance of the hSBA responses to the four primary MenB test strains to describe breadth of vaccine coverage is supported by the demonstration of protective bactericidal responses by MenB-FHbp also observed against diverse and contemporary MenB outbreak strains from Europe and the United States ${ }^{47,57}$ and against non-MenB disease-causing strains (ie, meningococcal serogroups $\mathrm{C}, \mathrm{Y}, \mathrm{W}$, and $\mathrm{X}$ ). ${ }^{58}$

Another methodology, the enzyme-linked immunosorbent assay-based Meningococcal Antigen Typing System (MATS), has been used to predict vaccine coverage of MenB-4C. ${ }^{59,60}$ However, MATS only predicts coverage of antigens specific to MenB-4C and is not useful for assessing coverage of other vaccines with different antigen compositions. ${ }^{47,61}$ Specifically, MATS measures 


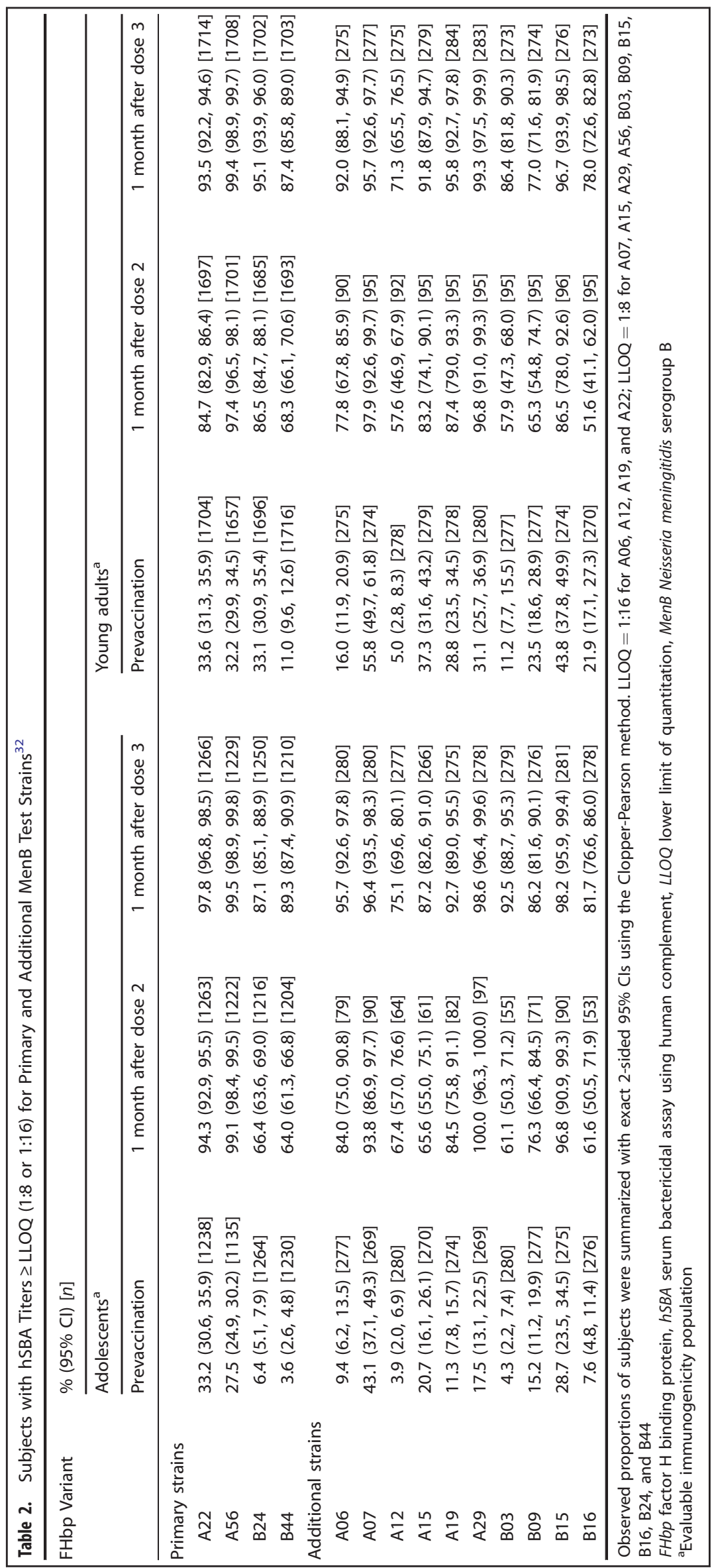




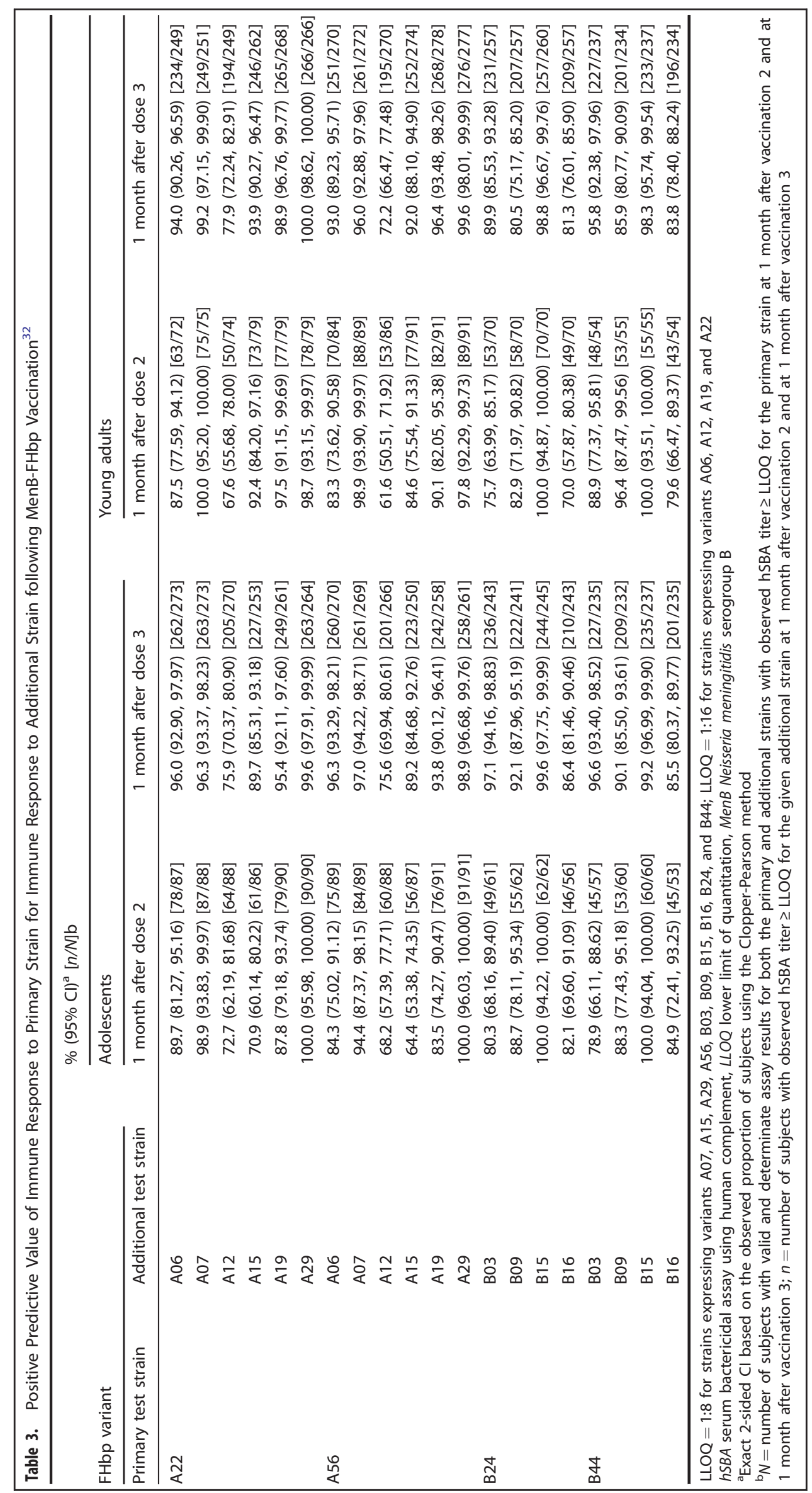


antigen expression rather than bactericidal activity and is reported as a relative potency compared with a reference strain for each antigen. ${ }^{61}$ If the relative potency for any one of the component antigens is commensurate with bactericidal activity for MenB-4C immune sera (i.e., achieves a positive bactericidal threshold), the strain is considered susceptible to killing. ${ }^{62}$ However, because sera from vaccinated individuals are not used in MATS, ${ }^{61}$ the assay is unable to predict the proportion of a population achieving hSBA titers $\geq 1: 4$ (i.e., the correlate of protection) in response to immunization.

Of note, limitations in performing hSBAs exist. ${ }^{47}$ For example, hSBAs are labor intensive and can require large quantities of sera and assay-compatible complement, particularly when larger numbers of strains and/or sera are to be assessed. In addition, interlaboratory differences in the performance of the assay reagents and strains used in hSBAs limit comparison of responses and assessments of breadth of coverage between vaccines. A known limitation of PPV analysis is the dependence of the magnitude of the response on prevalence (i.e., in this setting, the proportion of subjects achieving hSBA $\geq$ LLOQ for the additional strains). ${ }^{63}$ However, it is notable in this analysis that although there was a range of postvaccination responses to the additional strains (at 1 month postdose 2 and postdose 3), PPVs were uniformly high.

Taken together, the immunogenicity data obtained from the 10 additional MenB hSBA test strains support the response data obtained from the four primary MenB hSBA test strains and confirm the broad coverage of MenB isolates conferred by MenBFHbp. This work demonstrates the application of a rigorous assessment of a MenB vaccine's elicited immune response using the epidemiology of MenB strains with regard to the vaccine antigen sequence and expression, in conjunction with the recognized surrogate of protection (hSBA), and, using this knowledge, led to vaccine licensure.

\section{METHODS}

Quantitation of FHbp surface expression

For all strains, FHbp surface expression was quantified by the validated MEASURE assay, a flow cytometric assay using monoclonal antibody (MN86-994-11) recognition of a conserved FHbp epitope common to both FHbp subfamilies. ${ }^{27,35}$ Details of the MEASURE assay have been described previously. ${ }^{54}$ Briefly, $50 \mu \mathrm{L}$ of bacteria were plated per well of 96-well round-bottom polystyrene plates, then centrifuged and washed with $1 \%$ (weight/volume) bovine serum albumin (BSA) in $1 \mathrm{X}$ phosphate buffered saline (PBS). Monoclonal antibodies (MN86-994-11 [in-house antibody] or mouse IgG [negative control; Jackson Immunoresearch, Cat \#015-000-003, West Grove, PA, USA]) were added to the bacterial pellets to a final concentration of $6.7 \mu \mathrm{g} / \mathrm{mL}$ ( $335 \mathrm{ng} /$ well), resuspended, and incubated for $30 \mathrm{~min}$ on ice. Cells were washed twice in 1\% BSA in PBS followed by addition of biotinylated goat anti-mouse IgG (subclasses $1+2 a+2 b+3$; Jackson Immunoresearch, Cat \#115-065-165; $10 \mu \mathrm{g} / \mathrm{mL}, 500 \mathrm{ng} /$ well), resuspension, and incubation for $\mathbf{3 0}$ min on ice. After two washes, cells were resuspended in streptavidin-phycoerythrin (PE; BD Biosciences, Cat \#554061, San Jose, CA, USA; $5 \mu \mathrm{g} / \mathrm{mL}, 250 \mathrm{ng} /$ well). Cells were washed twice in $1 \%$ BSA in PBS and then resuspended in $1 \%$ paraformaldehyde. For each well, 20,000 events were acquired on an Accuri C6 flow cytometer and analyzed using Accuri CFlow software (BD Biosciences). The gating strategy of the MEASURE assay has been previously published..$^{54}$ For each sample, the mean fluorescence intensity (MFI) of the PE channel was determined after gating in the logarithmic forward scatter versus side scatter dot plot. FHbp expression was considered above the level of detection if MFIs were above an arbitrary threshold of at least 100 and three times that of the negative control (mouse lgG) MFI used in that assay. The cutoff level adopted for each FHbp variant group was the observed median mean fluorescence intensity plus 1 standard deviation, using the precision estimate of $25.2 \%$ relative standard deviation.
Immunogenicity analysis

Each of the 10 additional MenB test strains were used in hSBAs to test sera from subjects participating in 2 pivotal phase 3 studies of MenB-FHbp. ${ }^{32}$ A total of 900 subjects from each study were to be divided into three subsets ( $n=300$ each); the 10 additional test strains were allocated across these subsets so that two subsets each included 3 test strains and 1 subset included four test strains. The subsets included samples from 300 subjects to ensure that $\geq 150$ evaluable hSBA results from each study would be obtained. Immune responses measured by hSBA using phase 3 clinical study sera were based on the assay LLOQ, which was an hSBA titer equal to $1: 8$ or $1: 16$ depending on the strain.

\section{Positive predictive value analyses}

The PPV for each primary/additional strain pair within an FHbp subfamily was defined as the proportion of subjects responding to the additional strain (hSBA titer $\geq$ LLOQ for the additional strain) among the total number of primary strain responders (hSBA titer $\geq$ LLOQ for the primary strain). PPV analyses assessed whether observed hSBA responses to the four primary strains predicted immune responses to additional strains expressing FHbps from the same subfamily.

\section{Reporting summary}

Further information on research design is available in the Nature Research Reporting Summary linked to this article.

\section{DATA AVAILABILITY}

Upon request, and subject to certain criteria, conditions and exceptions (see https:// www.pfizer.com/science/clinical-trials/trial-data-and-results for more information), Pfizer will provide access to individual de-identified participant data from Pfizersponsored global interventional clinical studies conducted for medicines, vaccines and medical devices (1) for indications that have been approved in the US and/or EU or (2) in programs that have been terminated (i.e., development for all indications has been discontinued). Pfizer will also consider requests for the protocol, data dictionary, and statistical analysis plan. Data may be requested from Pfizer trials 24 months after study completion. The de-identified participant data will be made available to researchers whose proposals meet the research criteria and other conditions, and for which an exception does not apply, via a secure portal. To gain access, data requestors must enter into a data access agreement with Pfizer.

Received: 29 January 2019; Accepted: 20 November 2019; Published online: 29 January 2020

\section{REFERENCES}

1. MacNeil, J., Cohn, A. \& Centers for Disease Control and Prevention. in VPD Surveillance Manual Chapter 8, 1-11 (Centers for Disease Control and Prevention, Atlanta, GA, 2011).

2. Cohn, A. C. et al. Prevention and control of meningococcal disease: recommendations of the Advisory Committee on Immunization Practices (ACIP). MMWR Recomm. Rep. 62, 1-28 (2013).

3. Read, R. C. Neisseria meningitidis; clones, carriage, and disease. Clin. Microbiol. Infect. 20, 391-395 (2014).

4. Rosenstein, N. E., Perkins, B. A., Stephens, D. S., Popovic, T. \& Hughes, J. M Meningococcal disease. N. Engl. J. Med. 344, 1378-1388 (2001).

5. Stephens, D. S., Greenwood, B. \& Brandtzaeg, P. Epidemic meningitis, meningococcaemia, and Neisseria meningitidis. Lancet 369, 2196-2210 (2007).

6. Thompson, M. J. et al. Clinical recognition of meningococcal disease in children and adolescents. Lancet 367, 397-403 (2006).

7. Cohn, A. C. et al. Changes in Neisseria meningitidis disease epidemiology in the United States, 1998-2007: implications for prevention of meningococcal disease. Clin. Infect. Dis. 50, 184-191 (2010).

8. Centers for Disease Control and Prevention. Meningococcal Disease: Technical and Clinical Information. http://www.cdc.gov/meningococcal/clinical-info.html (2018).

9. Pace, D. \& Pollard, A. J. Meningococcal disease: clinical presentation and sequelae. Vaccine 30, B3-B9 (2012).

10. Harrison, L. H., Trotter, C. L. \& Ramsay, M. E. Global epidemiology of meningococcal disease. Vaccine 27, B51-B63 (2009).

11. Stefanelli, P. et al. Meningococci of serogroup X clonal complex 181 in refugee camps, Italy. Emerg. Infect. Dis. 23, 870-872 (2017). 
12. Agnememel, A. et al. Neisseria meningitidis serogroup $X$ in sub-Saharan Africa. Emerg. Infect. Dis. 22, 698-702 (2016).

13. Finne, J., Leinonen, M. \& Makela, P. H. Antigenic similarities between brain components and bacteria causing meningitis. Implications for vaccine development and pathogenesis. Lancet 2, 355-357 (1983).

14. Christodoulides, M. \& Heckels, J. Novel approaches to Neisseria meningitidis vaccine design. Pathog. Dis. 75, 1-16 (2017).

15. European Centre for Disease Prevention and Control. Surveillance Atlas of Infectious Diseases. https://ecdc.europa.eu/en/surveillance-atlas-infectious-diseases (2019).

16. Centers for Disease Control and Prevention. Enhanced Meningococcal Disease Surveillance Report 2016. https://www.cdc.gov/meningococcal/downloads/ NCIRD-EMS-Report.pdf (2018).

17. Public Health Agency of Canada. Vaccine Preventable Disease: Surveillance Report to December 31, 2015. https://www.canada.ca/en/public-health/services/ publications/healthy-living/vaccine-preventable-disease-surveillance-reportdecember-31-2015.html (2019).

18. Australian Government Department of Health. Invasive Meningococcal Disease National Surveillance Report With a Focus on MenW. https://www1.health.gov.au/ internet/main/publishing.nsf/Content/5FEABC4B495BDEC1CA25807D001327FA/ \$File/1Jan-31Mar2018-Consol-Invasive-Men-W.pdf (2018).

19. Institute of Environmental Science and Research Ltd. Notifiable Diseases in New Zealand: Annual Report 2016. https://surv.esr.cri.nz/PDF_surveillance/AnnualRpt/ AnnualSurv/2016/2016AnnualNDReportFinal.pdf (2018).

20. Lennon, D. et al. Reducing inequalities with vaccines: New Zealand's MeNZB vaccine initiative to control an epidemic. J. Paediatr. Child Health 48, 193-201 (2012).

21. Kelly, C., Arnold, R., Galloway, Y. \& O'Hallahan, J. A prospective study of the effectiveness of the New Zealand meningococcal B vaccine. Am. J. Epidemiol. 166, 817-823 (2007).

22. Martin, D. R., Ruijne, N., McCallum, L., O'Hallahan, J. \& Oster, P. The VR2 epitope on the PorA P1.7-2,4 protein is the major target for the immune response elicited by the strain-specific group B meningococcal vaccine MeNZB. Clin. Vaccin. Immunol. 13, 486-491 (2006).

23. Girard, M. P., Preziosi, M. P., Aguado, M. T. \& Kieny, M. P. A review of vaccine research and development: meningococcal disease. Vaccine 24, 4692-4700 (2006).

24. Wedege, E. et al. Functional and specific antibody responses in adult volunteers in New Zealand who were given one of two different meningococcal serogroup B outer membrane vesicle vaccines. Clin. Vaccin. Immunol. 14, 830-838 (2007).

25. Petousis-Harris, H. Impact of meningococcal group B OMV vaccines, beyond their brief. Hum. Vaccin. Immunother. 14, 1058-1063 (2018).

26. Fletcher, L. D. et al. Vaccine potential of the Neisseria meningitidis 2086 lipoprotein. Infect. Immun. 72, 2088-2100 (2004).

27. Zlotnick, G. W. et al. The discovery and development of a novel vaccine to protect against Neisseria meningitidis serogroup B disease. Hum. Vaccin. Immunother. 11, 5-13 (2015).

28. Madico, G. et al. The meningococcal vaccine candidate GNA1870 binds the complement regulatory protein factor $\mathrm{H}$ and enhances serum resistance. $J$. Immunol. 177, 501-510 (2006).

29. Masignani, V. et al. Vaccination against Neisseria meningitidis using three variants of the lipoprotein GNA1870. J. Exp. Med. 197, 789-799 (2003).

30. Welsch, J. A., Rossi, R., Comanducci, M. \& Granoff, D. M. Protective activity of monoclonal antibodies to genome-derived neisserial antigen 1870, a Neisseria meningitidis candidate vaccine. J. Immunol. 172, 5606-5615 (2004).

31. Bernfield, L. et al. Identification of a novel vaccine candidate for group B Neisseria meningitidis. in Proc. 13th International Pathogenic Neisseria Conference (Oslo, Norway, 2002).

32. Ostergaard, L. et al. A bivalent meningococcal B vaccine in adolescents and young adults. N. Engl. J. Med. 377, 2349-2362 (2017).

33. Murphy, E. et al. Sequence diversity of the factor $\mathrm{H}$ binding protein vaccine candidate in epidemiologically relevant strains of serogroup $B$ Neisseria meningitidis. J. Infect. Dis. 200, 379-389 (2009).

34. Trumenba ${ }^{\circledast}$ (meningococcal group $B$ vaccine). Full Prescribing Information (Wyeth Pharmaceuticals Inc (a subsidiary of Pfizer Inc), Philadelphia, PA, 2018).

35. Jiang, H. Q. et al. Broad vaccine coverage predicted for a bivalent recombinant factor $\mathrm{H}$ binding protein based vaccine to prevent serogroup $\mathrm{B}$ meningococcal disease. Vaccine 28, 6086-6093 (2010).

36. Trumenba ${ }^{\circledast}$ (MenB-FHbp). Summary of Product Characteristics (Pfizer Ltd, Sandwich, UK, 2018).

37. Australian Government Department of Health. Australian Register of Therapeutic Goods (ARTG). https://www.tga.gov.au/artg (2018).

38. Jackman, S. Political Science Computational Laboratory, version 1.5.2. https:// cran.r-project.org/web/packages/pscl/pscl.pdf (2018).

39. Bexsero (meningococcal group B vaccine). Full Prescribing Information (GSK Vaccines, Srl, Sovicille (SI), Italy, 2018).
40. Harris, S. L. et al. Neisseria meningitidis serogroup B vaccine, bivalent rLP2086, induces broad serum bactericidal activity against diverse invasive disease strains including outbreak strains. Pediatr. Infect. Dis. J. 36, 216-223 (2017).

41. Goldschneider, I., Gotschlich, E. C. \& Artenstein, M. S. Human immunity to the meningococcus. I. The role of humoral antibodies. J. Exp. Med. 129, 1307-1326 (1969).

42. Borrow, R. et al. Neisseria meningitidis group B correlates of protection and assay standardization-international meeting report Emory University, Atlanta, Georgia, United States, 16-17 March 2005. Vaccine 24, 5093-5107 (2006).

43. Frasch, C. E., Borrow, R. \& Donnelly, J. Bactericidal antibody is the immunologic surrogate of protection against meningococcal disease. Vaccine 27, B112-B116 (2009).

44. Sierra, G. V. et al. Vaccine against group B Neisseria meningitidis: protection trial and mass vaccination results in Cuba. NIPH Ann. 14, 195-207; discussion 208-110 (1991).

45. Milagres, L. G. et al. Immune response of Brazilian children to a Neisseria meningitidis serogroup B outer membrane protein vaccine: comparison with efficacy. Infect. Immun. 62, 4419-4424 (1994).

46. Holst, J. et al. Serum bactericidal activity correlates with the vaccine efficacy of outer membrane vesicle vaccines against Neisseria meningitidis serogroup B disease. Vaccine 21, 734-737 (2003).

47. Donald, R. G. et al. Meningococcal serogroup B vaccines: estimating breadth of coverage. Hum. Vaccin. Immunother. 13, 255-265 (2017).

48. Milagres, L. G., Gorla, M. C., Sacchi, C. T. \& Rodrigues, M. M. Specificity of bactericidal antibody response to serogroup B meningococcal strains in Brazilian children after immunization with an outer membrane vaccine. Infect. Immun. 66, 4755-4761 (1998).

49. Tappero, J. W. et al. Immunogenicity of 2 serogroup B outer-membrane protein meningococcal vaccines: a randomized controlled trial in Chile. JAMA 281, 1520-1527 (1999).

50. Tondella, M. L. et al. Distribution of Neisseria meningitidis serogroup B serosubtypes and serotypes circulating in the United States. The Active Bacterial Core Surveillance Team. J. Clin. Microbiol. 38, 3323-3328 (2000).

51. Holst, J. et al. Properties and clinical performance of vaccines containing outer membrane vesicles from Neisseria meningitidis. Vaccine 27, B3-B12 (2009).

52. Richmond, P. C. et al. Safety, immunogenicity, and tolerability of meningococcal serogroup B bivalent recombinant lipoprotein 2086 vaccine in healthy adolescents: a randomised, single-blind, placebo-controlled, phase 2 trial. Lancet Infect. Dis. 12, 597-607 (2012).

53. Richmond, P. C. et al. A bivalent Neisseria meningitidis recombinant lipidated factor $\mathrm{H}$ binding protein vaccine in young adults: results of a randomised, controlled, dose-escalation phase 1 trial. Vaccine 30, 6163-6174 (2012).

54. McNeil, L. K. et al. Predicting the susceptibility of meningococcal serogroup B isolates to bactericidal antibodies elicited by bivalent rLP2086, a novel prophylactic vaccine. MBio. 9, e00036-e00018 (2018).

55. Hoiseth, S. K. et al. A multi-country evaluation of Neisseria meningitidis serogroup $\mathrm{B}$ factor $\mathrm{H}$-binding proteins and implications for vaccine coverage in different age groups. Pediatr. Infect. Dis. J. 32, 1096-1101 (2013).

56. Borrow, R., Balmer, P. \& Miller, E. Meningococcal surrogates of protection-serum bactericidal antibody activity. Vaccine 23, 2222-2227 (2005).

57. Taha, M. K. et al. Bactericidal activity of sera from adolescents vaccinated with bivalent rLP2086 against meningococcal serogroup B outbreak strains from France. Vaccine 35, 1530-1537 (2017).

58. Harris, S. L. et al. The bivalent factor $\mathrm{H}$ binding protein meningococcal serogroup $B$ vaccine elicits bactericidal antibodies against representative non-serogroup $B$ meningococci. Vaccine 36, 6867-6874 (2018).

59. Vernikos, G. \& Medini, D. Bexsero ${ }^{\circledR}$ chronicle. Pathog. Glob. Health 108, 305-316 (2014).

60. Abad, R. et al. Predicted strain coverage of a new meningococcal multicomponent vaccine (4CMenB) in Spain: analysis of the differences with other European countries. PLOS ONE 11, e0150721 (2016).

61. Medini, D., Stella, M. \& Wassil, J. MATS: Global coverage estimates for 4CMenB, a novel multicomponent meningococcal B vaccine. Vaccine 33, 2629-2636 (2015).

62. Donnelly, J. et al. Qualitative and quantitative assessment of meningococcal antigens to evaluate the potential strain coverage of protein-based vaccines. Proc. Natl. Acad. Sci. USA 107, 19490-19495 (2010).

63. Altman, D. G. \& Bland, J. M. Diagnostic tests 2: predictive values. BMJ (Clin. Res. Ed.) 309, 102 (1994).

\section{ACKNOWLEDGEMENTS}

Editorial support was provided by Tricia Newell, PhD, of Complete Healthcare Communications, LLC (North Wales, PA), a CHC Group company, and was funded by Pfizer Inc. This work was funded by Pfizer Inc. 


\section{AUTHOR CONTRIBUTIONS}

S.H., K.J., A.S.A., and T.J. were involved in study conception and design. S.H., C.T., and T.J. were involved in acquisition of data. D.R. performed the statistical analysis. S.H., C.T., K.J., A.S.A., J.P., and T.J. were involved in analysis and interpretation of data. All authors participated in drafting of the manuscript or critically revising it for intellectual content. All authors approved the completed version of the manuscript and are accountable for all aspects of the manuscript content, including accuracy and integrity.

\section{COMPETING INTERESTS}

All authors are or were employees of Pfizer Inc. The authors declare that there are no other competing interests.

\section{ADDITIONAL INFORMATION}

Supplementary information is available for this paper at https://doi.org/10.1038/ s41541-019-0154-0.

Correspondence and requests for materials should be addressed to A.S.A.
Reprints and permission information is available at http://www.nature.com/ reprints

Publisher's note Springer Nature remains neutral with regard to jurisdictional claims in published maps and institutional affiliations.

(c) Open Access This article is licensed under a Creative Commons Attribution 4.0 International License, which permits use, sharing, adaptation, distribution and reproduction in any medium or format, as long as you give appropriate credit to the original author(s) and the source, provide a link to the Creative Commons license, and indicate if changes were made. The images or other third party material in this article are included in the article's Creative Commons license, unless indicated otherwise in a credit line to the material. If material is not included in the article's Creative Commons license and your intended use is not permitted by statutory regulation or exceeds the permitted use, you will need to obtain permission directly from the copyright holder. To view a copy of this license, visit http://creativecommons. org/licenses/by/4.0/.

(c) The Author(s) 2020 